\title{
Schwannoma of the vagina - a common tumor but a rare location: A case report
}

\author{
XIN AN ${ }^{1}$, MENG ZHU $^{2}$, NING ZHANG ${ }^{3}$, SANJUN LU $^{1}$, PIN WEI $^{1}$, LINNA JIANG ${ }^{1}$ and XUELI YANG ${ }^{1}$ \\ ${ }^{1}$ Department of Pathology, Handan First Hospital, Handan, Hebei 056002; ${ }^{2}$ Department of Gastroenterology, \\ First Affiliated Hospital of Xi'an Jiaotong University, Xi'an, Shaanxi 710061; ${ }^{3}$ Department of Pathology, \\ General Hospital of Ningxia Medical University, Yinchuan, Ningxia 750004, P.R. China
}

Received May 31, 2017; Accepted August 30, 2017

DOI: $10.3892 / \mathrm{mco} .2017 .1420$

\begin{abstract}
Schwannomas are nerve sheath tumors arising from Schwann cells. These tumors usually present as benign, relatively slow-growing, solitary, encapsulated, painless masses. Schwannomas rarely occur in the vagina, and have not been fully recognized as gynecological tumors. We herein describe the case of a patient who presented with a schwannoma occurring in the wall of vagina, with non-specific symptoms lasting for $\sim 1$ year. The vaginal mass was incidentally detected during a sonographic examination and the patient was referred for surgical resection. The surgery was uncomplicated and the vaginal tumor was diagnosed as benign schwannoma. The immunohistochemical examination revealed positivity for vimentin, S-100 and glial fibrillary acidic protein, whereas discovered on GIST-1, CD117, CD34, desmin, smooth muscle actin and cytokeratin were negative. Tumors occurring in the vagina are common and are of variable histological types, with a wide range of pathological characteristics and complications. Schwannoma should be considered in the differential diagnosis in patients presenting with atypical symptoms from the gynecological tract. Immunohistochemical staining is required for confirmation of the diagnosis of schwannoma, and for distinguishing this entity from other homologous tumors.
\end{abstract}

\section{Introduction}

Schwannoma (neurilemmoma) is a type of tumor that originates from Schwann cells, which produce the insulating myelin sheath covering peripheral nerves. Schwannomas are also

Correspondence to: Dr Meng Zhu, Department of Gastroenterology, First Affiliated Hospital of Xi'an Jiaotong University, 277 West Yanta Road, Xi'an, Shaanxi 710061, P.R. China E-mail: ricc_003@sina.com.cn

Abbreviations: SMA, smooth muscle actin; GFAP, glial fibrillary acidic protein; $\mathrm{CR}$, calretinin

Key words: schwannoma, vagina, immunohistochemistry, S-100 protein referred to as Schwann cell tumors and are benign, solitary, relatively slow-growing nerve sheath tumors (1). Theoretically, schwannomas may develop in any peripheral nerve. The most common site of schwannomas is the head and neck region, accounting for $25-35 \%$ of all cases (2). In 3-4\% of the patients, schwannomas of the head and neck are incidentally discovered at autopsy (3). Apart from cranial nerves, schwannomas may be observed in other sites, such as the tongue (4), stomach (5), colon (6), knee (7) and penis (8); however, it is highly unusual for schwannomas to be encountered in the genital tract.

Transformation into a malignant tumor is rarely encountered in schwannomas, and the reasons have not yet been fully elucidated. Schwannomas may transform into a type of malignant tumor referred to as neurofibrosarcoma.

The clinical manifestations of schwannomas differ according to tumor location and tumor size and they are mostly atypical. The majority of head and neck schwannomas are vestibular schwannomas, and tumors of the vestibulocochlear nerve may be associated with tinnitus and epicophosis. Complete surgical removal is considered to be the standard approach to the treatment of the vast majority of this type of tumor, due to its encapsulated nature (9).

We herein present a case of schwannoma located in the vagina in a 48-year-old woman, which was incidentally discovered.

\section{Case report}

A 48-year-old female patient, gravida 2 para 2, presented to the Handan First Hospital (Handan, China) in June 2016 with a main complaint of vaginal dilation persisting for $\sim 1$ year. The last menstrual period was in July 11, 2016. Apart from menstruation, the patient had not observed any vaginal bleeding or other vaginal discharge over the course of the disease. The findings on physical examination were as follows: Temperature $36.8^{\circ} \mathrm{C}$, heart rate 78 beats/min, respiratory rate 20 breaths $/ \mathrm{min}$, and blood pressure 125/80 $\mathrm{mmHg}$. Gynecological examination revealed a nodular mass located in the left posterior vaginal wall, in the upper part of the vagina. The size of the mass was $3.5 \times 2.5 \mathrm{~cm}$, and it was fixed (non-mobile), with a hard texture, clear boundary, smooth surface and irregular shape. The cervix, uterus and bilateral attachments did not exhibit any abnormalities on palpation. Transvaginal ultrasonography 

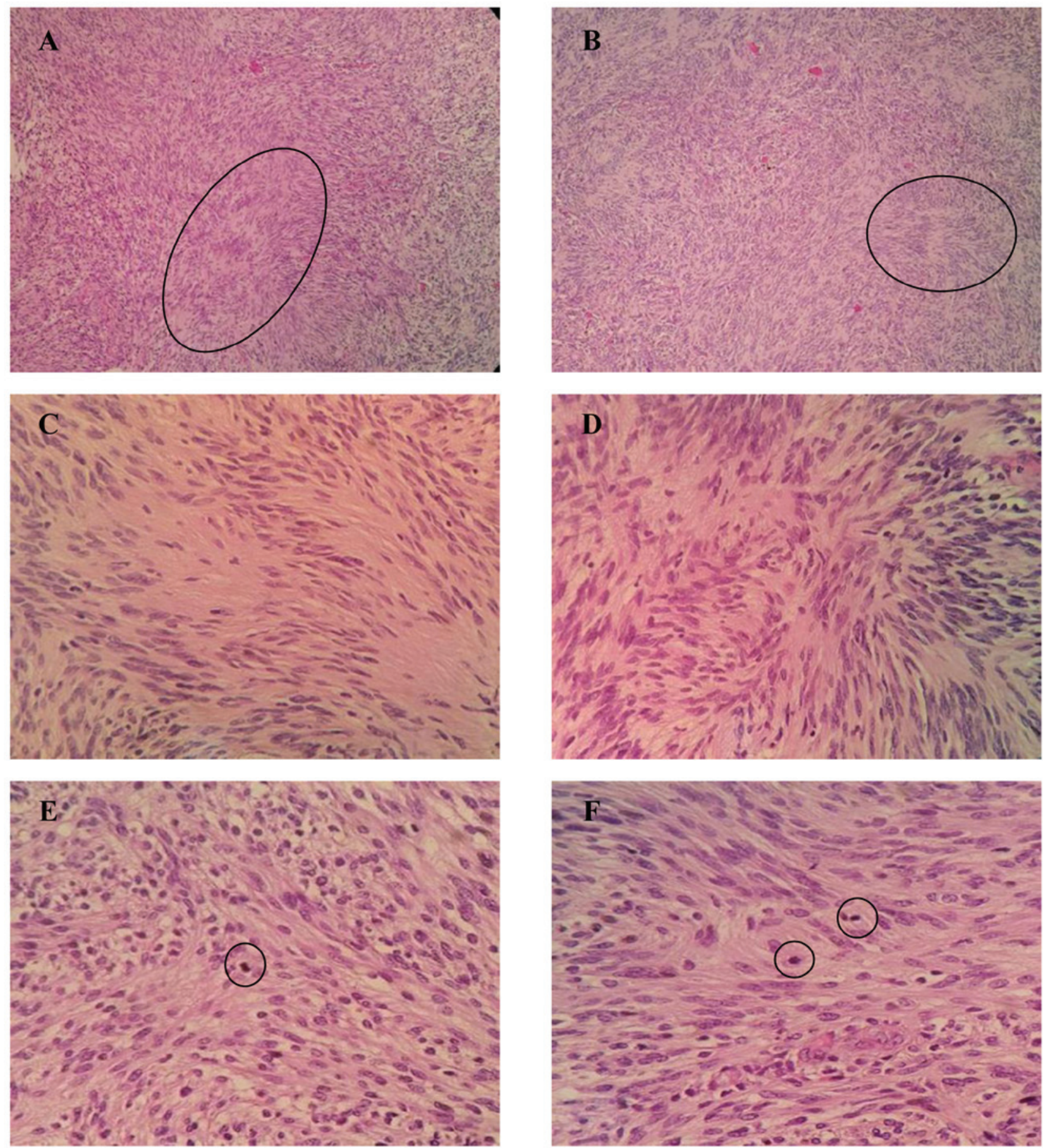

Figure 1. Hematoxylin and eosin staining of formalin-fixed and paraffin-embedded sections of the vaginal schwannoma. (A and B) Typical Antoni A hypercellular areas composed of spindle cells arranged in fascicular and sheet-like patterns, with nuclear palisading (circles). Original magnification, x100. (C and D) The tumor cells were long, spindle-shaped, with mild nuclear atypia. There were no necrotic areas. Original magnification, x200. (E and F) Mitotic figures were observed (circles). Original magnification, $x 400$.

revealed a low-echo mass on the left side of the vagina, sized $3.7 \times 2.7 \times 2.7 \mathrm{~cm}$. The liver and kidney function tests, chest X-ray and electrocardiogram were all normal. To determine the nature of the lesion, the patient underwent exploratory surgery through the transvaginal approach under epidural anesthesia. The intraoperative findings were as follows: The lesion was located in the left posterior wall of the vagina and it appeared to be nodular, consisting of three smaller fused nodules, sized $1 \times 0.5,1 \times 2$ and $0.5 \times 0.5 \mathrm{~cm}$. The mass had a clear boundary from the surrounding tissues, its surface was smooth and it was encapsulated. The mass was removed in a piecemeal manner and on cross-section the tumor exhibited yellowish areas and its texture was soft. On gross examination, the resected tissues were gray or grayish yellow in color, the size ranged from $0.5 \times 0.3 \times 0.2$ to $3.2 \times 1.5 \times 0.5 \mathrm{~cm}$, and the texture was soft and partly tough. On microscopic examination following hematoxylin and eosin (H\&E) staining, hypercellular and hypocellular areas (Antoni type A and B, respectively) with Verocay bodies were identified. The tumor cells were long, spindle-shaped, and mildly atypical, and were densely arranged in bundles, 

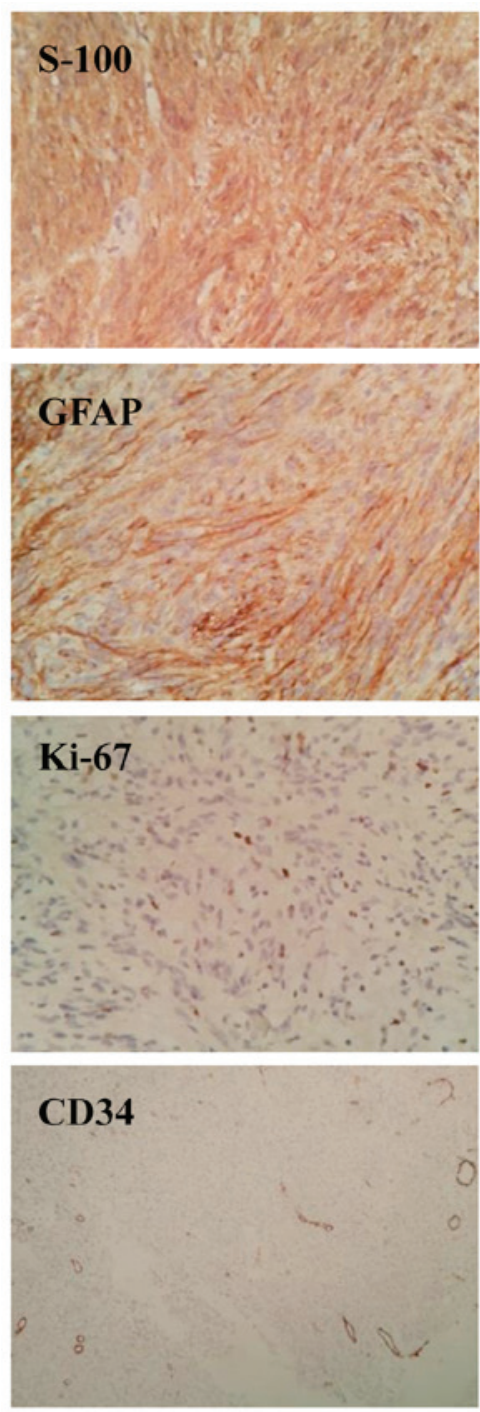
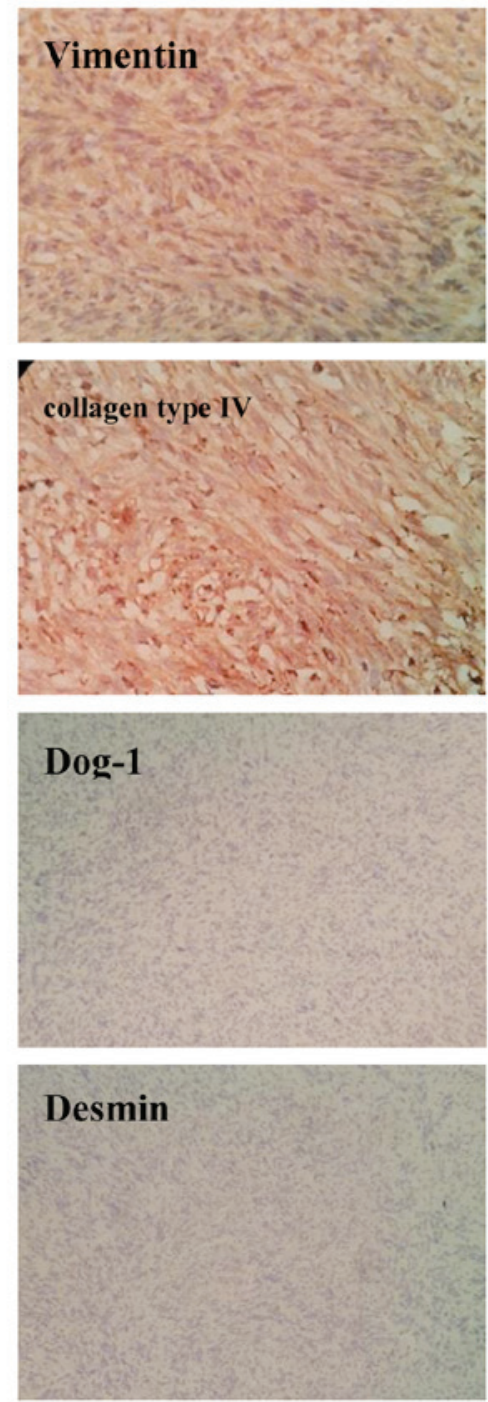
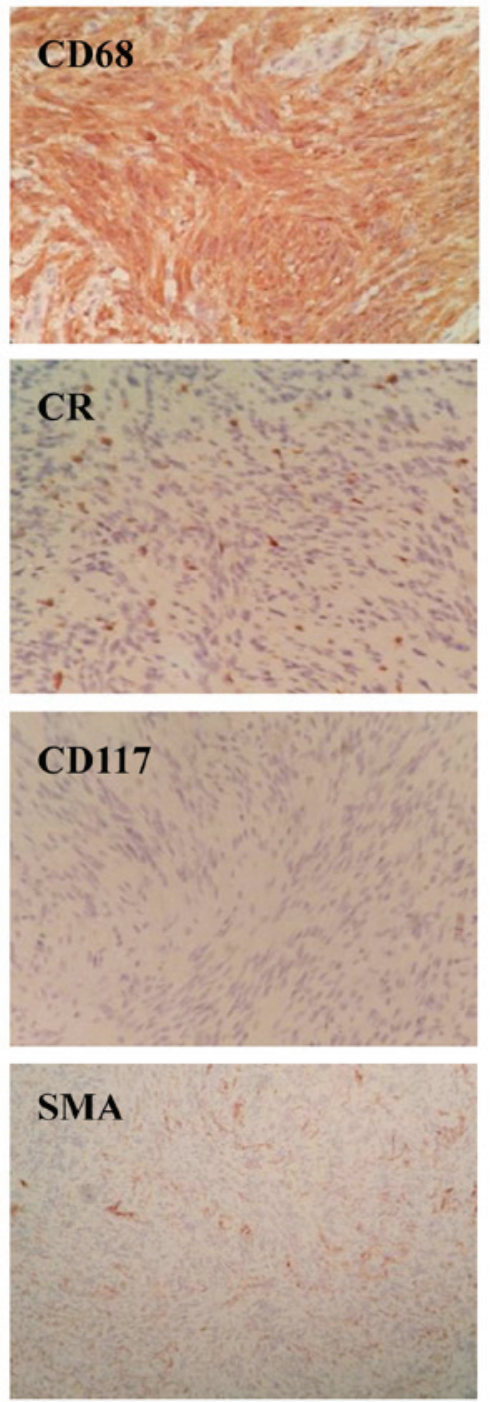

Figure 2. Immunostaining of the tumor section with hematoxylin counterstaining; original magnification, x200. The tumor cells were strongly positive for S-100, vimentin, CD68, GFAP and collagen type IV in Antoni A areas (3+, brownish-yellow cytoplasmic staining). CR was weakly positive (1+, nuclear staining). The Ki-67 proliferation index was 10\%. However, DOG-1, CD117, CD34 and desmin were all negative. SMA was positive in the vessels. GFAP, glial fibrillary acidic protein; CR, calretinin; DOG-1, discovered on GIST-1; SMA, smooth muscle actin.

palisades and whirlpools, with a mitotic count of 3/10 highpower fields (HPFs). No necrosis or mucoid degeneration was identified on microscopic examination. The histological appearance on H\&E staining is shown in Fig. 1. On immunohistochemical examination, the tumor cells were positive for vimentin (3+), S-100 (3+), CD68 (3+), glial fibrillary acidic protein (GFAP; $3+)$, collagen type IV $(3+)$, calretinin $(+)$ and Ki-67 (10\%); discovered on GIST-1 (DOG-1), CD117, CD34, CD99, desmin, smooth muscle actin (SMA) and cytokeratin were all negative. All immunohistochemistry reagents were purchased from Maxim Biotechnologies (Fuzhou, China). The immunohistochemical staining results are presented in Fig. 2. Taken together, these characteristics were consistent with a pathological diagnosis of benign cellular schwannoma. Due to the presence of active cell growth and mitotic figures, follow-up was recommended. The patient recovered quickly following complete margin-negative surgical resection, and there has been no recurrence or metastasis during the 1-year follow-up (last follow-up visit, May 2017).

\section{Discussion}

Cellular schwannoma, which is a benign peripheral nerve tumor with unknown histogenesis, has a predilection for the upper limbs and the head and neck region in children and young women (10). This type of tumor often grows slowly and is almost always solitary, encapsulated, circumscribed and eccentrically located. For vaginal schwannomas, early detection may be difficult due to the slow growth over time and unspecific clinical manifestations. The majority of the cases are asymptomatic and most of these tumors are incidentally discovered on imaging examinations (computed tomography, magnetic resonance imaging). Histologically, two main patterns, namely Antoni type A and B, are observed. Immunohistochemical examination is necessary for the definitive diagnosis of this type of tumor.

In the present case, the patient was a perimenopausal middle-aged woman. The symptoms were mild, without bleeding, pain or irritation, so the mass was not detected over 
a long period. A variety of clinical manifestations of vaginal schwannoma are reported, with late symptoms often occurring due to the increasing size of the tumor and depending on its location (11). Other clinical symptoms include vaginal pain when the tumor is large, and urinary retention and/ or constipation if the tumor compresses the bladder outlet and/or rectum, respectively (12-15). Our patient had a tumor measuring $\sim 3 \mathrm{~cm}$ and experienced a sensation of vaginal tenesmus. The diagnosis of this type of tumor relies on pathological examination of the surgically excised specimens revealing the classic schwannoma Antoni A and B areas. Antoni A areas are characteristically hypercellular, composed of spindle tumor cells (elongated Schwann cells) arranged in sheets, exhibiting a fascicular pattern and nuclear palisading arranged around a collagenous hyalinised core (Verocay bodies). The structure of Antoni type A areas is considered as a prominent characteristic for histopathological diagnosis. Antoni B areas are hypocelullar and are composed of small round cells within a myxoid stroma; the tumor cells in this area appear to be more disorganized compared with Antoni type $\mathrm{A}$ areas. This type is prone to cystic degeneration. In our patient, H\&E staining revealed that spindle cells were regularly arranged in whirlpools or palisades. The nuclear atypia was mild, and the mitotic count was 3/10 HPFs under the microscope. No necrosis or degenerative changes were observed. Therefore, the diagnosis of schwannoma may be made based on the histopathological characteristics described above. However, immunohistochemical staining is essential for confirming the diagnosis and distinguishing schwannoma from other homologous tumors. Schwannomas display strong and diffuse immunoreactivity for S-100 protein, vimentin and GFAP, and are negative for CD117, DOG-1, CD34 and SMA, which are findings suggestive of its neural origin. The patient in the present case received timely complete surgical resection and has had no recurrence during the follow-up period. Surgical resection was previously reported to be the only curative treatment for schwannoma $(9,16)$. As schwannomas are mostly benign with a good prognosis, aggressive surgery is not considered necessary.

Differential diagnosis: i) Leiomyoma is a benign tumor of smooth muscle (usually originating in the uterus or digestive tract). The tumor cells are spindle-shaped and are arranged in a weaving pattern. The nuclei are egg-shaped, round and blunt on both ends. Leiomyoma very rarely exhibits malignant transformation, except for cases where the nuclear mitotic count is 1-4/10 HPFs. On immunohistochemistry, the tumor cells are SMA-positive. ii) Interstitialoma is a benign tumor located in the digestive tract. The spindle cells form swirls and fascicles, with occasional palisading, and many have oval nuclei surrounded by perinuclear halos or vacuoles. On immunohistochemistry, the cells are CD117-, DOG-1- and CD34-positive. iii) Malignant schwannoma. There is a significant distinction between benign and malignant schwannomas. In malignant schwannomas, the tumor cells form clusters and may invade into the lumen of blood vessels. These tumors are highly cellular, with areas of necrosis commonly seen in the tumor parenchyma, and significant mitotic activity ( $\geq 4$ mitotic figures/10 HPFs). The expression of SOX10, neurofibromin and p16 is completely absent, epidermal growth factor receptor and S-100 may be weakly positive, and the
Ki-67 proliferation index is $>20 \%$ (17). iv) Neurofibroma is a peripheral nerve sheath tumor. The tumor is composed of slender spindle cells arranged in wavy, fascicular and circinate pattern, with collagen fibers or myxoid substance in the tumor stroma. The tumor lacks Antoni A and B areas and there is no calretinin expression. v) Solitary fibroma is a rare mesenchymal tumor composed of two parts, namely a hypercellular and a hypocellular region, in which collagen fibers exhibit unequal thickness and different shapes. The mitotic count is $<3 / 10$ HPFs. CD34, CD99, epithelial membrane antigen and B-cell lymphoma-2 are usually positive.

In conclusion, we herein present a rare case of vaginal schwannoma incidentally detected in a patient presenting only with mild discomfort. As this is a common disease, but a rare location, differential diagnosis of schwannoma from other vaginal tumors is crucial. Based on the histopathological and immunohistochemical characteristics, particularly staining for S-100, CD117, DOG-1, CD34, SMA and Ki-67, the diagnosis of schwannoma may be confirmed, even if the location of the tumor is unusual. Transvaginal surgical resection is curative, as in the present case. The recurrence rate and the risk of malignant transformation are exceptionally low in such cases.

\section{References}

1. Hilton DA and Hanemann CO: Schwannomas and their pathogenesis. Brain Pathol 24: 205-220, 2014.

2. Berlucchi M, Piazza C, Blanzuoli L, Battaglia G and Nicolai P: Schwannoma of the nasal septum: a case report with review of the literature. Eur Arch Otorhinolaryngol 257: 402-405, 2000.

3. Hanemann CO and Evans DG: News on the genetics. epidemiology, medical care and translational research of Schwannomas. J Neurol 253: 1533-1541, 2006.

4. Abreu I, Roriz D, Rodrigues P, Moreira A, Marques C and Alves FC: Schwannoma of the tongue-A common tumour in a rare location: A case report. Eur J Radiol Open 4: 1-3, 2017.

5. Romdhane H, Cheikh M, Mzoughi Z, Slama SB, Ennaifer R and Belhadj N: Gastric Schwannoma: A Case Report. Clin Pract 6: 849, 2016.

6. Wang WB, Chen WB, Lin JJ, Xu JH, Wang JH and Sheng QS: Schwannoma of the colon: A case report and review of the literature. Oncol Lett 11: 2580-2582, 2016.

7. Öz TT, Aktaş B, Özkan K, Özturan B, Kilic B and Demiroğlu M: A Case of Schwannoma of the Common Peroneal Nerve in the Knee. Orthop Rev (Pavia) 9: 6825, 2017.

8. Loeser A, Katzenberger T, Meuller JG, Riedmiller H and Gerharz EW: Solitary schwannoma of the glans penis. Urology 70: 1007.e5-1007.e6, 2007.

9. Biswas D, Marnane CN, Mal R and Baldwin D: Extracranial head and neck schwannomas--a 10-year review. Auris Nasus Larynx 34: 353-359, 2007.

10. Hornick JL and Fletcher CD: Cellular neurothekeoma: Detailed characterization in a series of 133 cases. Am J Surg Pathol 31: 329-340, 2007.

11. Terada S, Suzuki N, Tomimatsu N and Akasofu K: Vaginal schwannoma. Arch Gynecol Obstet 251: 203-206, 1992.

12. Obeidat BR, Amarin ZO and Jallad MF: Vaginal schwannoma: A case report. J Reprod Med 52: 341-342, 2007.

13. Park JW, Hwang SO, Choi SJ, Lee BI, Park JH and Song E: Incidental diagnosis of vaginal schwannoma in a patient with thigh pain. Obstet Gynecol Sci 57: 86-88, 2014.

14. Sharma NS and Lynch MJ: Intrapelvic neurilemmoma presenting with bladder outlet obstruction. Br J Urol 82: 917, 1998.

15. Samarakoon L, Weerasekera A, Sanjeewa R and Kollure S: Giant presacral schwannoma presenting with constipation: A case report. J Med Case Reports 6: 285, 2012.

16. Melvin WS and Wilkinson MG: Gastric schwannoma. Clinical and pathologic considerations. Am Surg 59: 293-296, 1993.

17. Pekmezci M, Reuss DE, Hirbe AC, Dahiya S, Gutmann DH, von Deimling A, et al: Morphologic and immunohistochemical features of malignant peripheral nerve sheath tumors and cellular schwannomas. Mod Pathol 28: 187-200, 2015. 